\section{Zum Einfluss sozialer Strukturen von Schulklassen auf schulisches Problemverhalten}

Thomas Begert, Christoph Müller, Gérard Bless

Universität Freiburg/Schweiz

\section{Problemstellung}

Im schulischen Alltag belasten Lehrpersonen weniger die seltenen und extremen Formen von Dissozialität (z.B. physische Gewalt) als die häufiger auftretenden, milderen Ausprägungen schulischen Problemverhaltens (z.B. Schwatzen oder Umhergehen während des Unterrichts usw.). Diese Verhaltensweisen vollziehen sich im Unterricht im Interaktionsfeld zwischen Lehrperson und Schüler/innen (vgl. Müller u.a. 2012) und gehen für Kinder und Jugendliche häufig mit negativen leistungsbezogenen und sozialen Konsequenzen einher.

Während Literatur und Praxis zur Erklärung schulischen Problemverhaltens vor allem individuums- und lehrpersonenzentrierte Faktoren betrachten, liegt der Schwerpunkt dieses Dissertationsprojekts auf der Analyse des Einflusses der Peerbeziehungen in der Schulklasse.

Sozialökologische Kontexte stellen, wie durch Schulklima- und Gewaltforschung aufgezeigt wurde, ein wichtiges Ursachenfeld für schulische Verhaltensschwierigkeiten dar. So spielen Interaktionen und Beziehungen unter Gleichaltrigen hinsichtlich der Verhaltensentwicklung eine wichtige Rolle (Howe 2010). Ein häufig diskutierter Peereinflussmechanismus ist in diesem Zusammenhang der Gruppendruck. Dieser ist umso größer, je stärker der Gruppenzusammenhalt ist (Cartwright 1968). Merkmale der sozialen Struktur von Schulklassen könnten daher einen Einfluss auf individuelle, unterrichtsbezogene Verhaltensschwierigkeiten ausüben.

\section{Forschungsstand}

Zur Beschreibung der sozialen Strukturen von Gruppen wird häufig der Begriff der „Kohäsion“ verwendet. Gruppenkohäsion wird in der sozialpsychologischen Forschung dabei vielfältig defi- niert und erfasst (vgl. Hogg 1992; Evans/Jarvis 1980). Ein mögliches Verständnis von Kohäsion stellt das Ausmaß der gegenseitigen affektiven Präferenzen der Gruppenmitglieder dar (Shaw 1981). Diese lassen sich beispielsweise soziometrisch durch die Anzahl oder Nähe sozialer Beziehungen zwischen Akteuren erfassen (Friedkin 2004).

Grundsätzlich sprechen mehrere empirische Befunde für positive Zusammenhänge zwischen Kohäsion und erwünschtem Verhalten (z.B. Leistung, Motivation, Kooperation, Wohlbefinden usw. Levine/Moreland 1990; Forsyth 2006). Entsprechend fand Meier (2009), dass eine höhere Klassenkohäsion mit weniger individuellem unterrichtsbezogenem Problemverhalten einherging.

Diese direkte Beziehung könnte sich jedoch, gemäß anderer Studien zur Gruppenkohäsion, auch komplexer gestalten. So wäre es unter bestimmten Bedingungen auch denkbar, dass eine hohe Kohäsion das Ausmaß abweichenden Verhaltens in einer Gruppe verstärkt: Je höher die Gruppenkohäsion, desto einheitlicher und ausgeprägter erscheinen die vorherrschenden Gruppennormen, was zu einem erhöhten Druck in Richtung eines bestimmten Verhaltens führt (Colman/Caron 2001). Gruppennormen repräsentieren dabei regulative Verhaltensstandards und Erwartungen zwischen Gruppenmitgliedern (Feldman 1984). In einer kohäsiven Klasse mit einer schuldistanzierten Klassennorm würde ein erhöhter Konformitätszwang dann zu mehr individuellem schulischem Problemverhalten beitragen. Soziale Kohäsion könnte also, je nach Richtung der sozialen Normen, ein zweischneidiges Schwert sein (Langfred 1998). Es ist deshalb zu prüfen, inwiefern die Klassennormen die Beziehung zwischen Klassenkohäsion und schulischem Problemverhalten moderieren.

Entsprechend erster Befunde von Xie und Johns (2000) könnte es aber auch sein, dass die Gruppenkohäsion erst die Gruppennormen hervorruft, die dann wiederum das individuelle Verhalten formen. So weisen Klassen mit proschulischen Normen mehr Beziehungsdichte, weniger Cliquen und eine geringere Anzahl sozial isolierter Schüler/innen auf (Specht/Fend 1979). Grup- 
pennormen wiederum beeinflussen individuelles Verhalten (Parks 2004; Hackman 1976). Es ist daher zusätzlich zu klären, inwiefern ein Effekt der Kohäsion auf individuelles schulisches Problemverhalten durch die Klassennormen mediiert wird.

\section{Fragestellungen}

1. Welchen (direkten) Effekt hat die Kohäsion von Schulklassen auf individuelles schulisches Problemverhalten?

2. Wird der Effekt der Kohäsion auf individuelles schulisches Problemverhalten durch die Klassennormen moderiert?

3. Wird der Effekt der Kohäsion auf individuelles schulisches Problemverhalten durch die Klassennormen mediiert?

\section{Methode der Vorstudie}

Der in dieser Forschungsarbeit analysierte Datensatz stammt aus der „Freiburger Studie zum Peereinfluss in Schulen“ (FRI-PEERS, Müller/Bless 2013) sowie einer Vorstudie davon (Müller u.a. 2013). Die Stichprobe der Vorstudie umfasste dabei 659 Schülerlinnen aus 35 Schulklassen der Stufen 7-9 aus verschiedenen Kantonen der deutschsprachigen Schweiz. Die Erhebungen fanden in einem anonymisierten Selbstauskunftsverfahren statt. Schulisches Problemverhalten wurde anhand der „Freiburger Selbst- und Peerauskunftsskalen“ (Müller u.a. 2012) gemessen. Zur Erhebung der Klassenstruktur wurden Interaktionspräferenz-Nominationen eingesetzt. Dies begründet sich in einem relationalen Verständnis sozialer Struktur, welches sich durch netzwerk-bzw. graphentheoretische Maßeinheiten der Kohäsion repräsentieren lässt (Wasserman/Faust 2009). Die Kohäsion wurde dabei als „Clustering Coefficient" (Newman u.a. 2002) mithilfe des sozialen Netzwerkprogramms Pajek bestimmt. Der Clustering Coefficient beschreibt hier, vereinfacht ausgedrückt, das proportionale Verhältnis zwischen der Anzahl geschlossener Dreierbeziehungen (,Der Freund meines Freundes ist auch mein Freund") zu allen Dreierbeziehungen in einer Klasse. Die deskriptive Klassennorm wurde als Mittelwert schulischen Problemverhaltens in der Klasse definiert (Chang 2004).
Die Moderator- und Mediatoranalysen, gemäß Urban und Mayerl (2008), erfolgten mithilfe des Statistikprogramms SPSS.

\section{Erste Ergebnisse der Vorstudie}

Regressionsanalysen zeigten einen kleinen, aber höchstsignifikant negativen Effekt der Klassenkohäsion auf schulisches Problemverhalten $(B=$ $\left.-0.15 ; R^{2}=.02 ; p<.001\right)$. Je höher also die Kohäsion in einer Klasse war, desto weniger individuelles schulisches Problemverhalten wurde berichtet. Dies stimmt mit den Ergebnissen von Meier (2009) überein.

Es fanden sich keine Interaktionseffekte zwischen Kohäsion und Klassennorm ( $p>$.05), was gegen die These einer moderierenden Rolle der Klassennorm spricht.

In Bezug auf die Mediatoranalyse wies der direkte Pfad von der Kohäsion auf die Klassennorm einen höchstsignifikant negativen Effekt auf $(B=-0.44$; $\mathrm{R}^{2}=.17 ; \mathrm{p}$ (.001). Dies bedeutet, dass eine höhere Klassenkohäsion mit schulfreundlicheren Klassennormen einherging. Je ausgeprägter die Klassennorm zugunsten schulischen Problemverhaltens ausfiel, desto mehr individuelles schulisches Problemverhalten wurde berichtet $\left(B=0.39 ; R^{2}=.15\right.$; $\mathrm{p}<.001)$. Dieser indirekte Effekt war entsprechend des Sobel-Tests (vgl. Preacher/Hayes 2004) höchstsignifikant $\left(R^{2}=.15 ; p<.001\right)$, und der Prädiktor „Klassenkohäsion“ verlor unter Einbezug der Klassennorm seine Signifikanz. Diese Ergebnisse sprechen insgesamt dafür, dass der Effekt der Kohäsion komplett durch die Klassennormen interveniert wurde. Dies bestätigt sich auch bei einer negativbinomialen Modellierung, die aufgrund der rechtschiefen Verteilung der individuellen Verhaltensdaten zusätzlich geprüft wurde (Hilbe 2008).

\section{Ausblick}

Die ersten Ergebnisse der Vorstudie deuten darauf hin, dass die Klassenkohäsion, in Beziehung mit den vorherrschenden Klassennormen, individuelles schulisches Problemverhalten beeinflussen könnte. Um differenziertere Aussagen zu solchen Effekten machen zu können, sollen die 
aufgeworfenen Fragen im Rahmen der Hauptstudie weiterverfolgt werden. Dazu wird ein Längsschnittdatensatz mit Befragungen von $825 \mathrm{Ju}$ gendlichen der 7. Klasse mehrebenenanalytisch ausgewertet werden.

Weitere Informationen und Literaturangaben können eingeholt werden bei thomas.begert@ unifr.ch

\section{Unterricht gestalten für Schülerinnen und Schüler mit Verhaltensstörungen im integrativen Setting}

\section{Ein Forschungsprojekt an der Inter- kantonalen Hochschule für Heilpädagogik zu Gelingensbedingungen für die Integration von Schülerinnen und Schülern mit Verhaltensstörungen}

\author{
Margaretha Florin, Annette Lütolf Bélet, \\ Angela Wyder \\ $\mathrm{HfH}$ Zürich
}

\section{Forschungshintergrund}

Störungen des Unterrichts, Konflikte mit Mitschülerinnen und Mitschülern, Provokationen: viele Verhaltensweisen von Schülerinnen und Schülern mit Verhaltensstörungen stellen für Lehrpersonen eine Herausforderung und nicht selten eine Belastung dar. Es stellt sich den Lehrpersonen die Frage, wie der Unterricht zu gestalten sei, um diese Kinder und Jugendlichen zu mehr aufgabenbezogenem Lernen zu führen.

\section{Forschungsfragen}

Die konkrete Ausgestaltung des Unterrichts für Schülerinnen und Schüler mit Verhaltensstörungen ist in der empirischen Forschung wenig untersucht worden. Das hier vorgestellte Forschungsprojekt soll einen Beitrag leisten zur Frage, wie der Unterricht in methodisch-didaktischer sowie in interaktiver Hinsicht gestaltet werden soll, damit Schülerinnen und Schüler mit Verhaltensstörungen im integrativen Setting vermehrt aufgabenbezogenes Verhalten zeigen und der ganzen Klasse ein störungsfreies Lernen ermöglicht wird.

\section{Forschungsdesign}

Die Stichprobe umfasste 40 Schülerinnen und Schüler mit externalisierenden Verhaltensstörungen aus zwanzig Regelklassen der Mittelstufe der Primarschule des Kantons Zürich.

Es wurden systematische Unterrichtsbeobachtungen nach der Time-Sampling-Methode (Textor 2007) durchgeführt.

Die verwendeten Beobachtungsbogen enthielten folgende Dimensionen:

Bereich Methodik: Unterrichtmethoden, Sozialformen, Differenzierung, Klarheit und Strukturiertheit, Unterstützung des Lernprozesses

Bereich Klassenmanagement: Haltung der Lehrperson, Kontrolle des Unterrichtsgeschehens, Verstärkung, Art von Interventionen

Ebene Schülerinnen und Schüler mit Verhaltensstörungen: aufgabenbezogenes Verhalten, störendes Verhalten.

\section{Kurzzusammenfassung der Ergebnisse}

\section{Deskription des Unterrichts}

a) Methodisch-didaktische Aspekte:

Insgesamt kann festgestellt werden, dass ein eher lehrerzentrierter Unterricht beobachtet wurde. So wurde in etwas mehr als der Hälfte der beobachteten Zeiteinheiten an von der Lehrperson gegebenen schriftlichen Aufgaben gearbeitet. Weiter machten mündliche Arbeitsformen wie das Unterrichtsgespräch und der Lehrervortrag zusammen etwa einen Viertel der Unterrichtszeit aus.

Offene Unterrichtsformen konnten in einem Fünftel der Unterrichtszeit beobachtet werden. Bewegungspausen fanden selten statt.

Die Arbeit im Klassenverband war die am häufigsten eingesetzte Sozialform, gefolgt von Einzelarbeit. Kooperative Formen fanden in rund einem Fünftel der beobachteten Unterrichtszeit statt. 
In rund $40 \%$ der Zeit wurde der Unterricht differenziert. Am häufigsten wurde eine quantitative Differenzierung bezüglich Aufgabenmenge bzw. Lerntempo beobachtet. Weiter wurde qualitativ häufig nach Aufgabeninhalt differenziert, seltener nach Schwierigkeitsgrad, Medien/Material und Lernweg.

Organisatorische Differenzierungen nach Arbeitsort und Sozialform konnten ebenfalls recht häufig beobachtet werden.

In einem differenzierten Unterricht wurden überwiegend zwei oder mehr Differenzierungsformen angewendet. In knapp der Hälfte der Zeit kamen sogar drei oder mehr (bis zu sieben) Formen gleichzeitig zur Anwendung.

Zur Gewährleistung von Klarheit und Strukturiertheit wurden von den Lehrpersonen am häufigsten die Ziele und Inhalte genannt, das Zeitfenster wurde viermal weniger angegeben.

In mehr als der Hälfte der Unterrichtszeit konnte eine Unterstützung des Lernprozesses durch die Lehrpersonen beobachtet werden. Am häufigsten bestand diese im Erklären und Modellieren sowie im Feedback Geben. Weitere Unterstützungsformen waren Hinweise geben, Fortschritte evaluieren und sehr selten die Anregung der Metakognition.

\section{b) Interaktive Aspekte:}

Die Haltung der Lehrpersonen war überwiegend sachorientiert. Eine auffallend durch Wertschätzung geprägte Haltung konnte selten und eine durch Ungeduld geprägte Haltung sehr selten beobachtet werden. Beinahe durchgehend hatten die Lehrpersonen das Unterrichtsgeschehen unter Kontrolle und nutzten die Unterrichtszeit für die Auseinandersetzung mit dem Lerninhalt. Nur in einem Prozent der Unterrichtszeit hatten die Lehrpersonen den Überblick verloren.

Lediglich in einem Zehntel der Unterrichtszeit konnten Verstärkungen beobachtet werden. Interventionen und Methoden der Störungskontrolle konnten gut dreimal häufiger beobachtet werden als Verstärkungen. Am meisten setzten Lehrpersonen dazu kurze Ermahnungen ein. Seltener waren nonverbale Störungskontrollen und Zurechtweisungen vor der Klasse.

\section{Zusammenhänge zwischen dem Unterricht und dem Lern- und Arbeitsverhalten der Schülerinnen und Schüler mit Verhaltensstörungen}

Die vorliegende Studie zeigt auf, dass verschiedene Bereiche der methodisch-didaktischen Gestaltung sowie der interaktiven Elemente des Unterrichts einen Zusammenhang mit dem Lernund Arbeitsverhalten der Schülerinnen und Schüler aufweisen.

Vermehrt aufgabenbezogenes Verhalten wurde beobachtet bei Einzelarbeiten, Bewegungspausen sowie bei einer Differenzierung nach Schwierigkeitsgrad. Bei kooperationsoffenen Einzelarbeiten (also wenn freigestellt wurde, ob alleine oder zu zweit gearbeitet wird) sowie bei häufiger Störungskontrolle der Lehrperson trat aufgabenbezogenes Verhalten seltener und störendes Verhalten häufiger auf. Störendes Verhalten wurde außerdem vermehrt während Stationen-, Werkstatt- oder Wochenplanarbeit beobachtet sowie beim gleichzeitigen Einsatz von mehreren Differenzierungsformen. Bei verbalen Unterstützungen während Lernprozessen trat störendes Verhalten vermehrt zutage.

Eher weniger störend verhielten sich die Schülerinnen und Schüler bei vorgegebenen Aufgaben, Projekt- bzw. Freiarbeit und Bewegungspausen, bei Partnerarbeit, dem Unterricht im Klassenverband und während des Einsatzes von Strategien zur Zielklarheit.

Das zudem berechnete logistische Regressionsmodell erklärt mit dem Einschluss der Variablen zur methodisch-didaktischen und interaktiven Unterrichtsgestaltung beim störenden Verhalten $17 \%$ der Varianz. Beim aufgabenbezogenen Verhalten sind es $10 \%$.

\section{Fazit und Ausblick}

Thesenartig werden hier einige Schlussfolgerungen aus dem Forschungsprojekt gezogen, welche für Lehrpersonen handlungsleitend sein können.

1. „Einfache“ und gut organisierte Arrangements sind zu bevorzugen: Einzel- und (gut organisierte) Partnerarbeiten sowie regelmäßige Bewegungspausen erhöhen das aufgabenbezogene Verhalten. \begin{tabular}{l|l} 
VHN $4 \mid 2014$
\end{tabular} 
2. Differenzierung ja, aber in Maßen: Differenzierung (v.a. inhaltliche Differenzierung) begünstigt das aufgabenbezogene Verhalten, jedoch nur, wenn nicht mehrere Differenzierungsformen gleichzeitig angewendet werden.

3. Ziele transparent machen: Ein durch Zielklarheit gekennzeichneter Unterricht führt zu weniger störendem Verhalten.

4. Lehrpersonen stören gelegentlich ihren eigenen Unterricht: Häufige Interventionen zur Störungskontrolle können aufgabenbezogenes Verhalten verringern und störendes erhöhen.

5. Unterstützungsleistungen sparsam und gezielt einsetzen: Auch mit Unterstützungsleistungen können die Schülerinnen und Schüler in ihrem Denkprozess unterbrochen werden. Sie sollen darum gezielt an einzelne Adressaten gerichtet werden statt als Inputs an die ganze Klasse.

Die vorliegende Studie hat explorativen Charakter und bezieht sich auf eine relativ kleine Stichprobe. Weiterführende Forschungsprojekte könnten die Tiefenstruktur des Unterrichts fokussieren und beispielsweise mittels Videoanalysen die hier gefundenen Zusammenhänge genauer erforschen.

Weitere Informationen und Literaturhinweise können eingeholt werden bei annette.luetolf@ hfh.ch

\section{SToRCH+: Simulationstraining mit dem RealCare ${ }^{\circledR}$ Baby - Schweiz}

\section{Dagmar Orthmann Bless}

Universität Freiburg/Schweiz

$\mathrm{SToRCH}^{+}$ist ein simulationsbasiertes Bildungsprogramm für Jugendliche und junge Erwachsene beiderlei Geschlechts ab ca. 16 Jahren. Dabei versorgen die Teilnehmenden das RealCare ${ }^{\circledR}$ Baby - einen computergestützten Simulator - während mehrerer Tage selbstständig. In begleitenden pädagogischen Sequenzen reflektieren sie das Erlebte und erweitern Wissen und Handlungskompetenzen in Bezug auf die eigene Lebensbewältigung.

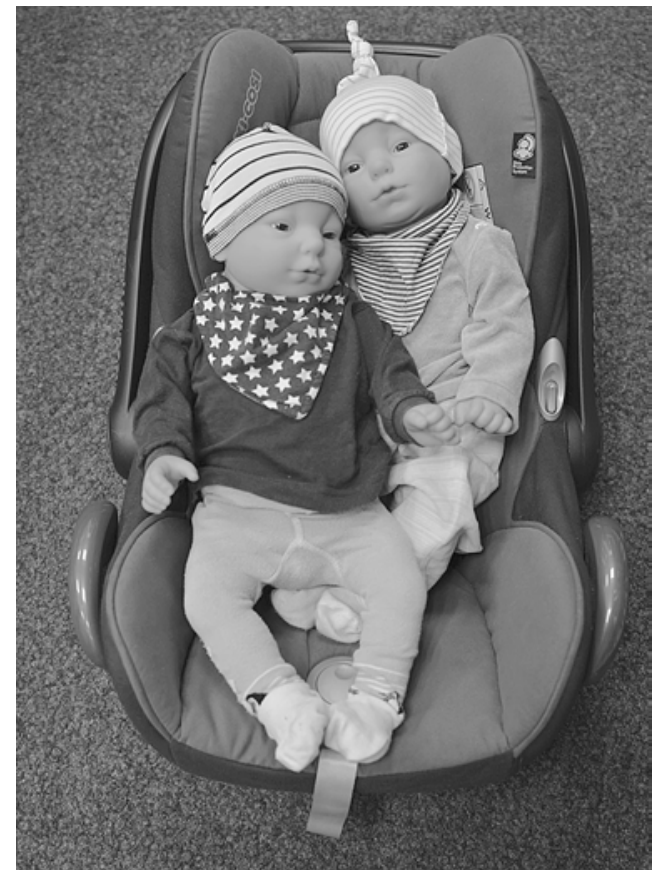

Die Adaptation des Programms an die Lernvoraussetzungen und Bedürfnisse von jungen Menschen mit Lernbeeinträchtigungen im Sinne einer Lernbehinderung oder geistigen Behinderung ist Gegenstand eines Forschungsprojektes, welches von der Schweizerischen Eidgenossenschaft, Büro für die Gleichstellung von Menschen mit Behinderungen, gefördert wird. Wir berichten an dieser Stelle über die Ziele und den Arbeitsprozess des Forschungsprojektes.

\section{Ausgangslage und Zielsetzungen}

Das Simulationstraining mit dem RealCare ${ }^{\circledR}$ Baby ist bereits in ca. 50 Ländern der Welt etabliert. Es dient Jugendlichen zur Auseinandersetzung mit alterstypischen Entwicklungsaufgaben im weiteren Sinne sowie zur Auseinandersetzung mit der verantwortungsvollen Aufgabe der Elternschaft im engeren Sinne. Die Pflicht, sich mit alterstypischen Entwicklungsaufgaben auseinanderzusetzen und dabei entsprechende Selbstkompetenzen für die eigene Lebensbewältigung zu entwickeln, haben alle jungen Menschen. Auch das Recht, sich als Persönlichkeit frei entfalten 
zu dürfen, sowie das Anrecht auf Bildung, die notwendig ist, um dieses Recht wahrnehmen zu können, schließt alle Menschen - unabhängig von ihren individuellen Lernvoraussetzungen mit ein. Junge Menschen mit Beeinträchtigungen konnten bisher vom Simulationstraining mit dem RealCare ${ }^{\circledR}$ Baby kaum profitieren, weil es in der vorliegenden Form ihren Lernvoraussetzungen nicht hinreichend entsprach. Eine Anpassung des Programms an die Bedürfnisse dieser speziellen Zielgruppe ist deshalb das zentrale Anliegen des Forschungsprojektes.

Bei der Analyse des internationalen Status Quo zur Arbeit mit dem RealCare ${ }^{\circledR}$ Baby wurden zudem folgende zwei Desiderate ermittelt, die auch für die Anwendung im sog. Standardbereich bestehen:

- Die zentrale Innovation der Arbeit mit dem RealCare ${ }^{\circledR}$ Baby ist das Simulationslernen. Es unterscheidet dieses Programm von anderen Ansätzen zur Unterstützung der Persönlichkeitsentwicklung. Allerdings hat bisher keine programmspezifische Auseinandersetzung mit dieser Form des experimentellen Lernens und den damit verknüpften Möglichkeiten und Vorteilen stattgefunden.

- Eine weitere Lücke besteht im Hinblick auf die Evaluation des Programms: Es fehlen bisher Möglichkeiten, Lerneffekte und Wirkungen systematisch zu dokumentieren.

\section{Arbeitsprozess}

Die Weiterentwicklung bestehender Arbeitsformen mit dem RealCare ${ }^{\circledR}$ Baby im Hinblick auf diese drei Aspekte wurde bzw. wird innerhalb unseres Forschungsprojektes folgendermaßen umgesetzt:

\section{Erarbeitung der lerntheoretischen Grundlagen}

a) Simulationslernen im pädagogischen Kontext: situative Bedingungen; Vorteile aus lernpsychologischer, ökonomischer und ethischer Perspektive; Grenzen

b) Lernvoraussetzungen und Bedürfnisse von jungen Menschen mit Lernbeeinträchtigungen im Sinne einer Lernbehinderung oder geistigen Behinderung c) Aspekte einer notwendigen Programmanpassung im Hinblick auf die identifizierten Möglichkeiten und Voraussetzungen

2. Entwicklung einer adaptierten Form des Simulationstrainings $\left(\mathrm{SToRCH}^{+}\right)$unter Berücksichtigung der Lernvoraussetzungen beeinträchtigter junger Menschen in Bezug auf:

a) Adressaten und Anwendungssituationen

b) Zielsetzungen und Lernmöglichkeiten

c) Lernarrangements und Hilfestellungen

d) Spezielle pädagogische Sequenzen

e) Arbeitsmaterialien und Evaluationsinstrumente

3. Erprobung von $\mathrm{SToRCH}+$ in der Praxis:

a) bei verschiedenen Alterskohorten (Jugendliche, Erwachsene)

b) in unterschiedlichen Anwendungssituationen (Gruppentrainings, Einzeltrainings)

c) mit unterschiedlichen Zielstellungen (Auseinandersetzung mit jugendtypischen Entwicklungsaufgaben, Realitätsprüfung eines bestehenden Kinderwunsches)

d) unter Anwendung verschiedener, eigens entwickelter Evaluationsinstrumente für verschiedene Bereiche (Wissen, praktische Kompetenzen in Handling und Care, emotionale Belastung, Motivation)

4. Überarbeitung des Programms in Bezug auf die unter 2. genannten Gesichtspunkte

5. Erarbeitung eines Programmhandbuches inkl. Arbeitsmaterialien für Fachpersonen

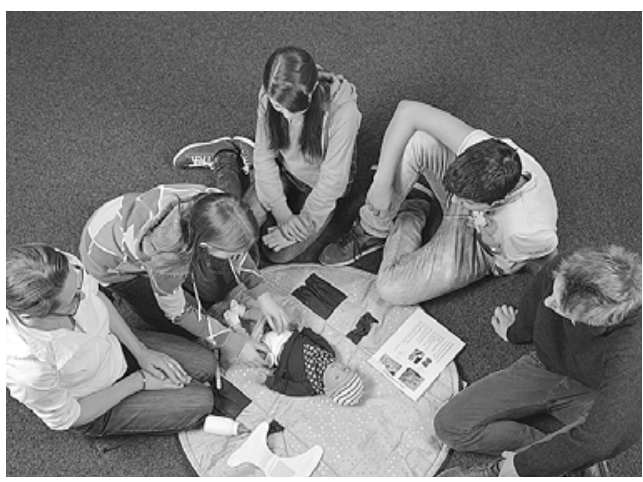

$\mathrm{VHN}_{4} \mid 2014$ 343 


\section{Zwischenbilanz und Ausblick}

Bei der praktischen Erprobung erzielte das adaptierte Programm eine hohe Akzeptanz sowohl bei Teilnehmenden als auch bei Fachpersonen. Die Evaluationsinstrumente erwiesen sich als praktikabel. Die wissenschaftliche Auswertung erhobener Daten zur Performanz verschiedener Teilnehmergruppen während des Simulationstrainings erfolgt in Kürze.

Die adaptierte Programmversion $\mathrm{SToRCH}+$ Simulationstraining mit dem RealCare ${ }^{\circledR}$ Baby - Schweiz erscheint im September 2014 als Handbuch. Das Handbuch in Verbindung mit Materialien und Kopiervorlagen (im Download-Bereich auf der Webseite) ermöglicht Fachpersonen aus
Pädagogik, Sonderpädagogik, Sozialpädagogik, Erwachsenenbildung, Sexualpädagogik und verwandten Fachgebieten eine den Qualitätsstandards entsprechende Planung und Durchführung eigener SToRCH+-Projekte in Schulen, Wohneinrichtungen oder sozialpädagogischen Settings.

Fachlichen Support für Anwender vor Ort stellen wir weiterhin u. a. in Form von Anwenderschulungen (universitär oder Inhouse) zur Verfügung.

Weitere Informationen zum Projekt und Literaturangaben können eingeholt werden bei dagmar.orthmann@unifr.ch

Programminformationen: http://fns.unifr.ch/sepial de 